\title{
Predicting the probability of meningioma recurrence based on the quantity of peritumoral brain edema on computerized tomography scanning
}

\author{
Ross E. Mantle, M.D., Boleslaw Lach, M.D., Ph.D., Mauricio R. Delgado, M.D., Salleh Baeesa, \\ M.D., and Gerard Bélanger, M.D.
}

Divisions of Neurosurgery and Neurology and Departments of Laboratory Medicine and Neuroradiology, Ottawa Hospital, Civic Campus, and University of Ottawa, Ottawa, Ontario, Canada

Object. The goal of this study was to determine whether the quantity of peritumoral brain edema displayed on computerized tomography (CT) scanning could be correlated with brain invasion and subsequent recurrence of meningiomas.

Methods. One hundred thirty-five patients who underwent resection of intracranial meningiomas at the Ottawa Civic Hospital were followed during the period 1980 to 1998. A complete resection was defined as one in which tumor, invaded bone, and involved dura were removed. Tumors were examined microscopically for evidence of brain invasion. The mean follow-up period was 9 years ( \pm 4 years, standard deviation [SD]) and the mean time to recurrence was 5 years ( \pm 4 years, SD). The authors used a simple grading system based on the average thickness (in centimeters) of edema seen on an axial CT slice showing the most tumor.

Edema grade was linearly related to edema volume determined by digitizing the scans $(\mathrm{r}=0.96 ; 29$ cases). The chance of brain invasion increased by $20 \%$ for each centimeter of edema $\left(r_{s}=1, p<0.0001\right.$; 124 cases). The presence of brain invasion was predictive of recurrence after complete resection with an accuracy of $83 \%$, a sensitivity of $89 \%$, and a specificity of $82 \%$. The chance of recurrence within 10 years after complete resection was given by the equation: percentage chance of recurrence $=$ (centimeter of edema $)^{3} \mathrm{X} 0.7$, which can be used to predict the chance of recurrence based on findings on CT scans $\left(\mathrm{r}_{\mathrm{s}}=1.00, \mathrm{p}<0.0001 ; 86\right.$ patients $)$. Statistical significance was confirmed using Kaplan-Meier and univariate and multivariate analyses. Completeness of resection was the most powerful predictor of recurrence $(\mathrm{p}<0.00001, \mathrm{r}=0.6)$, followed by edema grade and brain invasion (both $\mathrm{p}=0.02, \mathrm{r}=0.1$ ). Patient age and gender and tumor location, size, and histological subtype were nonsignificant factors.

Conclusions. Brain invasion causes peritumoral edema. Invaded brain tissue is also the source of residual cells in cases of tumor recurrence after gross-total resection.

Key Words * meningioma * peritumoral edema * tumor recurrence * Simpson grade * blood-brain barrier 
Meningiomas are slow-growing, well-circumscribed tumors that arise from the arachnoid cap cells of the dura mater. They may occur at any dural site in the central nervous system and in the ventricles, but are most commonly located in the supratentorial intracranium. Meningiomas typically have a broad base of attachment to the dura. They may invade or cause hyperostosis of subjacent bone. They usually indent and sometimes invade the brain. Gross-total resection of a meningioma is associated with an expectation of permanent cure and is frequently a source of satisfaction to neurosurgeons and their patients.

Unfortunately, despite gross-total resection with removal of involved dura and bone, approximately $10 \%$ of patients experience tumor recurrence.[2] This often leads to reoperations and much greater risk of morbidity and mortality for the patient.[9] It is believed that recurrences represent the continued proliferation of tumor cells left behind after resection; however, the anatomical location of the reservoir of these residual cells has not been conclusively established.

Previous studies, including the unpublished observations of one of us (M.R.D.) and the recent work of Bitzer, et al.,[5] support an association between brain invasion by meningiomas and peritumoral edema observed on computerized tomography (CT) scans. Our objective in the present study was to determine whether the quantity of peritumoral edema could be correlated with both brain invasion by tumor and subsequent tumor recurrence.

\section{CLINICAL MATERIAL AND METHODS}

An observational (noninterventional), prospective follow-up study was conducted over a period of 19 years from 1980 through 1998. The study was comprised of 135 patients, who were consecutively treated by the staff of the Ottawa Civic Hospital adult neurosurgical service, in whom preoperative CT scans had been obtained, and for whom a diagnosis of intracranial meningioma had been made based on pathological findings at surgery. Laboratory pathological data, CT scans, and clinical and operative records were examined. In cases of multiple tumors occurring simultaneously or serially in a single patient, each operative resection was considered as a separate case for the purpose of analysis. Patients who died postoperatively before leaving the hospital were not included in the follow-up time calculations. Peritumoral edema data were available in 124 cases.

\section{Completeness of Resection}

Resections were divided into complete and incomplete groups. A complete resection was deemed to have occurred when all three of the following criteria were met: 1) gross-total resection in the surgeon's opinion as recorded in the operative note (this criterion was thought to be necessary because some small residual tumor visible at surgery may be too small to be visualized by the resolution provided by present-generation CT scanners or magnetic resonance [MR] imagers); 2) excision, not cauterization, of involved dura and excision or autoclave sterilization of invaded bone; and 3) no evidence of residual tumor on postoperative CT or MR images. Removal of hyperostotic bone without gross evidence of invasion was not required for a complete resection.

\section{Follow-Up Examination for Recurrence}

The endpoint for recurrence was a CT or MR image consistent with meningioma in a location contiguous with the previous resection. Patients at our institution underwent yearly follow-up imaging for 4 years, after which they were followed via clinic visits or by telephone interview in cases in which no recent clinic visits were made. Recent CT scans were available in 10 of the 77 nonrecurrent cases followed 
beyond 4 years, none of which revealed recurrent tumor. All living patients interviewed by telephone reported that they were free of symptoms referable to brain tumor, and all relatives of patients who died during the follow-up period reported that death had occurred from unrelated causes. Follow-up times were calculated from the time of surgery to the time at which either the patient died, was lost to follow-up review, or the study ended.

\section{Edema Grading System}

Grading of edema on CT scans was performed by a single neuroradiologist (G.B.) who had no knowledge of the pathological results. Edema was graded by means of a simple scale in common, informal use by neurosurgical residents to quantify peritumoral edema. The edema grade is expressed as the thickness of the low-attenuation area (7-25 Hounsfield units), measured to the nearest centimeter from the outer edge of the tumor to the outer edge of edematous parenchyma on the best axial slice (that which shows the largest cross-sectional area of tumor). Because edema thickness may vary about a tumor, this measurement was taken at a point subjectively thought to represent the average edema thickness (Fig. 1). The centimeter scale shown on the CT scan was used to scale the measurement of edema. This grading scale was interpreted nonparametrically for the purpose of statistical analysis.

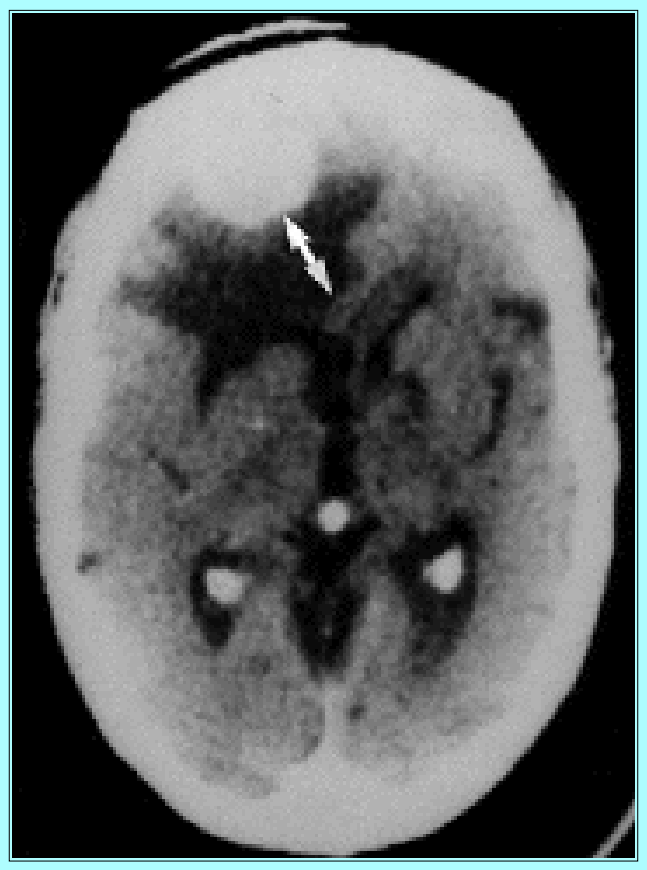

Fig. 1. Computerized tomography scan providing an example of a right frontal meningioma with edema Grade 2. The thickness of edema is measured radially from the edge of the tumor to the outer margin of the low-attenuation area (7-25 Hounsfield units) as indicated by the white double-tipped arrow. A point of average edema thickness on the axial slice that contains the most tumor is chosen. The measurement is scaled to centimeters by using the scale provided on the scan.

\section{Validation of the Edema Grading System}

Our edema grading scale, based on subjectively determined average radial centimeters, was validated by correlation with the actual volume of edema determined by digitizing 29 CT scans and by using public domain software (Image version 1.60; National Institutes of Health, Bethesda, MD) to calculate edema volume and tumor volume in cubic centimeters. 


\section{Histopathological Study}

Determination of the presence of microscopic invasion and histological typing of tumors was made by a single neuropathologist (B.L.) without knowledge of the edema grade. Invasion of the tumor into the brain parenchyma was deemed present when any of the following criteria were met after we performed a standard sampling protocol: 1) a hematoxylin and eosin-stained sample of the brain-tumor interface was available showing invasion of tumor into the brain (Fig. 2 upper); 2) a sample of tumor was available containing brain tissue within it that displayed reactive astrocytosis or neuronal degeneration (Fig. 2 center); or 3) reactive astrocytes within the tumor were revealed using standard glial fibrillary acidic protein (GFAP) immunostaining (Fig. 2 lower).

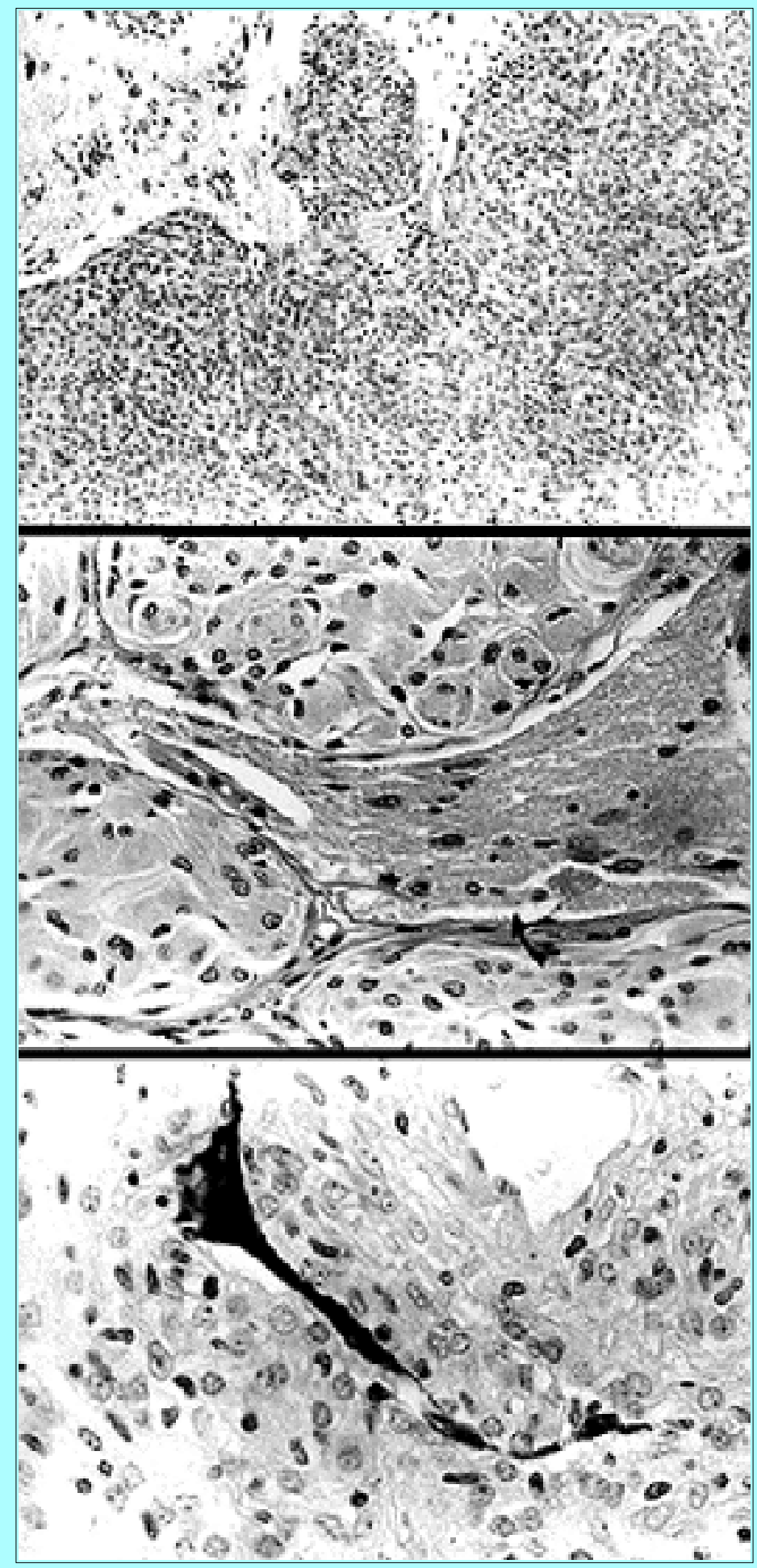


Fig. 2. Photomicrographs of histopathological specimens that meet criteria for brain invasion. Upper: Meningioma (bottom) and brain (top) interface showing invasion of a perivascular tongue of tumor into the brain parenchyma. $\mathrm{H} \& \mathrm{E}$, original magnification $\mathrm{X}$ 400. Center: Meningioma with entrapped brain tissue (curved arrow) showing reactive astrocytosis and neuronal degeneration. H \& E, original magnification X 1000. Lower: A GFAP-positive astrocyte within a meningioma. Original magnification X 1600.

Tumors were divided into histological subtypes on the basis of World Health Organization criteria. Tumor location was classified as left or right sided, parasagittal, frontal, planum sphenoidale, occipital, temporal, convexity, sphenoid wing, perisellar, cerebellopontine angle, tentorial, intraventricular, or falx.

\section{Statistical Analysis}

Statistical analysis was performed using commercially available computer software (SPSS for Windows; Cary, SC). Parametric and nonparametric linear regression, baysian predictive values, Kaplan-Meier survival analysis, logistic regression, and Cox univariate and multivariate models were used.

\section{RESULTS}

The average patient age at the time of enrollment was $72 \pm 11$ years (standard deviation [SD]) and the male/female ratio was 1:2. The overall percentage of recurrences was $32 \%$, and the recurrence rate after complete resection was $14 \%$. Complete resection of $69 \%$ of tumors was achieved. The percentage of recurrences after incomplete resection was $76 \%$. The mean time to recurrence after complete resection was $5 \pm 4$ years (SD), and the mean follow-up time was $9 \pm 4$ years (SD). The percentages of tumors by location and histological subtype are given in Table 1 . There were nine perioperative deaths and no intraoperative deaths. In $48 \%$ of all cases peritumoral edema was evident. 


\begin{tabular}{|c|c|}
\hline \multicolumn{2}{|c|}{$\begin{array}{c}\text { TABLE } 1 \\
\text { PERCENTAGE OF MEN NGOMA CASES BV LOCATION AND } \\
\text { HISTOLOGICAL SUBTYPE }\end{array}$} \\
\hline Factor & Percentage of Cases \\
\hline \multicolumn{2}{|l|}{ location } \\
\hline It side & 50 \\
\hline rt side & 50 \\
\hline frontal & 31 \\
\hline convexity & 16 \\
\hline parasagittal & 16 \\
\hline sphenoid wing & 13 \\
\hline planum sphenoidale & 9 \\
\hline perisellar & 7 \\
\hline cerebellopontine angle & 4 \\
\hline occipital & 3 \\
\hline tentorial & 3 \\
\hline in traventricular & 2 \\
\hline tem poral & $<1$ \\
\hline fal $x$ & $<1$ \\
\hline \multicolumn{2}{|l|}{ histological subtype } \\
\hline meningothelial & 35 \\
\hline transitional & 33 \\
\hline transitional \& meningothelial & $<1$ \\
\hline vacuolated & 5 \\
\hline fitrous & 4 \\
\hline fitrous \& psammomatous & 1 \\
\hline fitrous \& transitional & $<1$ \\
\hline cellular & 3 \\
\hline cellular \& meningothelial & $<1$ \\
\hline psammomatous & 2 \\
\hline secretory' & 1 \\
\hline syncytial & 1 \\
\hline suncitial \& transitional & $<1$ \\
\hline vascular & 1 \\
\hline cystic & $<1$ \\
\hline microcystic \& secretory & $<1$ \\
\hline pleiomorphic & $<1$ \\
\hline atypical & 3 \\
\hline malignant & 5 \\
\hline
\end{tabular}

Malignant varieties (World Health Organization Grades II-IV) comprised 8\% of the cases (13 tumors, of which eight were malignant and five were atypical). The male/female ratio for cases of malignant tumor was 2.7:1, and the mean age of the patients was 77 years (range 68-86 years). All cases for which data were available were associated with peritumoral edema. One patient died perioperatively. Seven patients experienced recurrence; in three of these the tumor recurred after incomplete resection. Forty percent of cases in which there was recurrence after complete resection involved tumor of the malignant subtypes.

The mean patient age at the time of resection in cases in which there was recurrence ( $72 \pm 10$ years [SD]) was not significantly different from that in cases in which there was no recurrence ( $73 \pm 11$ years [SD]; $p$ $=0.69)$. The initial volume of tumors that recurred $\left(39 \pm 45 \mathrm{~cm}^{3}[\mathrm{SD}]\right)$ was not significantly different from the volume of those that did not recur $\left(50 \pm 59 \mathrm{~cm}^{3}[\mathrm{SD}] ; \mathrm{p}=0.35\right)$.

Evidence of brain invasion by tumor was predictive of the chance of recurrence after complete resection: $36 \%$ of tumors with invasion recurred and $1 \%$ of tumors without invasion recurred; that is, the positive predictive value (PPV) of invasion for recurrence was $36 \%$ and the negative predictive value (NPV) of 
absence of invasion for recurrence was $99 \%$, with an odds ratio of 36 . The overall accuracy of invasion as a predictor of recurrence was $83 \%$, the sensitivity was $89 \%$, and the specificity was $82 \%$ (Fig. 3).

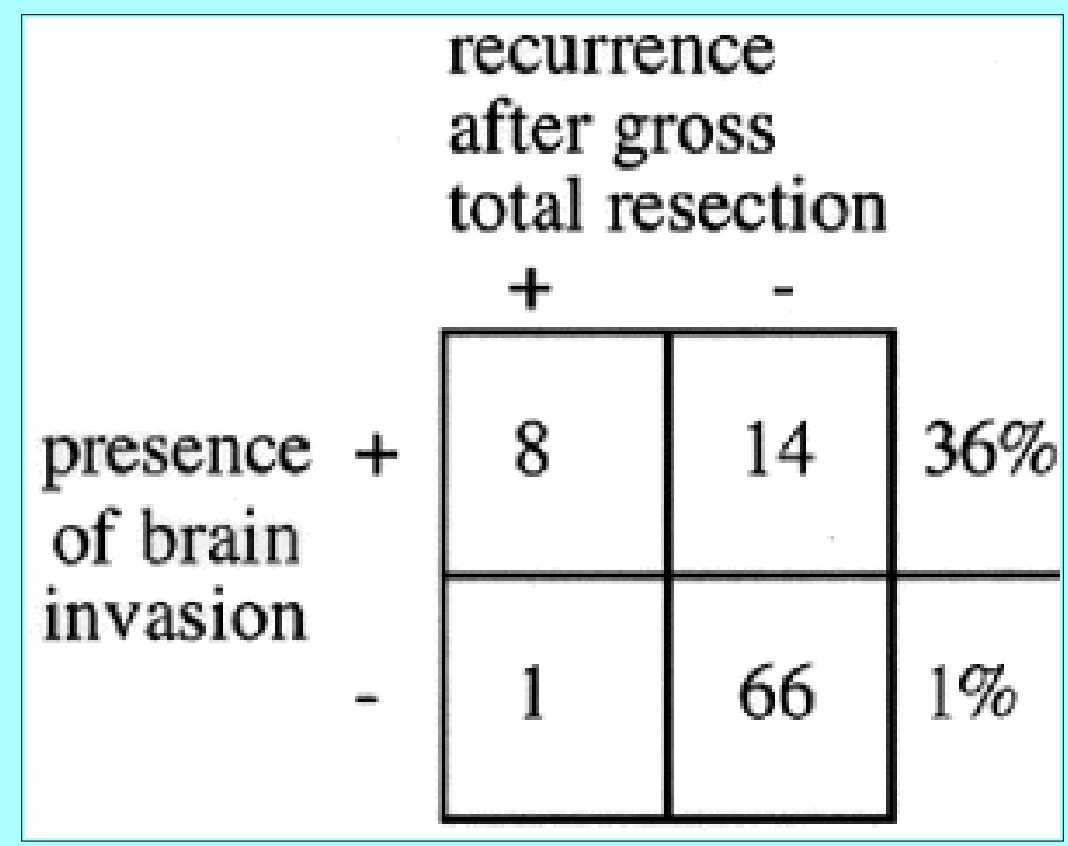

Fig. 3. Chart showing that brain invasion by meningiomas predicts recurrence after complete resection. Sensitivity $=89 \%$, specificity $=82 \%, \mathrm{PPV}=36 \%, \mathrm{NPV}=99 \%$, odds ratio $=36$.

The peritumoral edema grade correlated linearly with edema volume. Edema volume (in cubic centimeters) was equal to 33 multiplied by the edema grade (in centimeters) ( $r=0.96,29$ cases; Fig. 4).

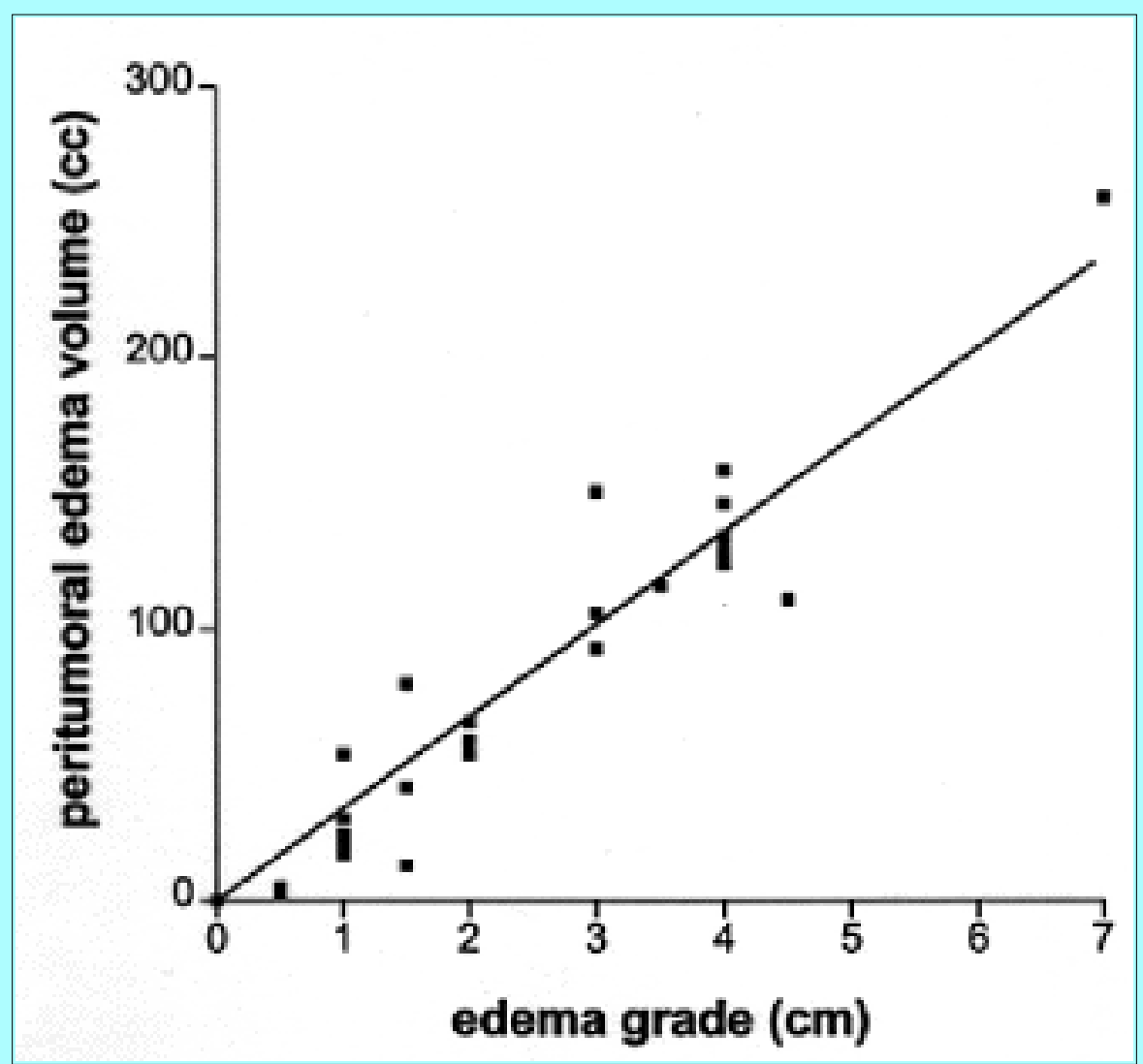

Fig. 4. Graph validating the edema grading method. Edema grade is compared with edema 
volume as measured by digitizing $\mathrm{CT}$ scans. The regression equation approximates: edema volume $=33 \mathrm{X}$ edema grade (in centimeters) $(r=0.96,29$ cases $)$.

The peritumoral edema grade correlated linearly with the chance of brain invasion (that is, the proportion of tumors showing brain invasion). The chance of invasion increased by approximately $20 \%$ for each centimeter of edema (Spearman's rank correlation coefficient $r_{s}=1.0, p<0.0001$; Fig. 5).

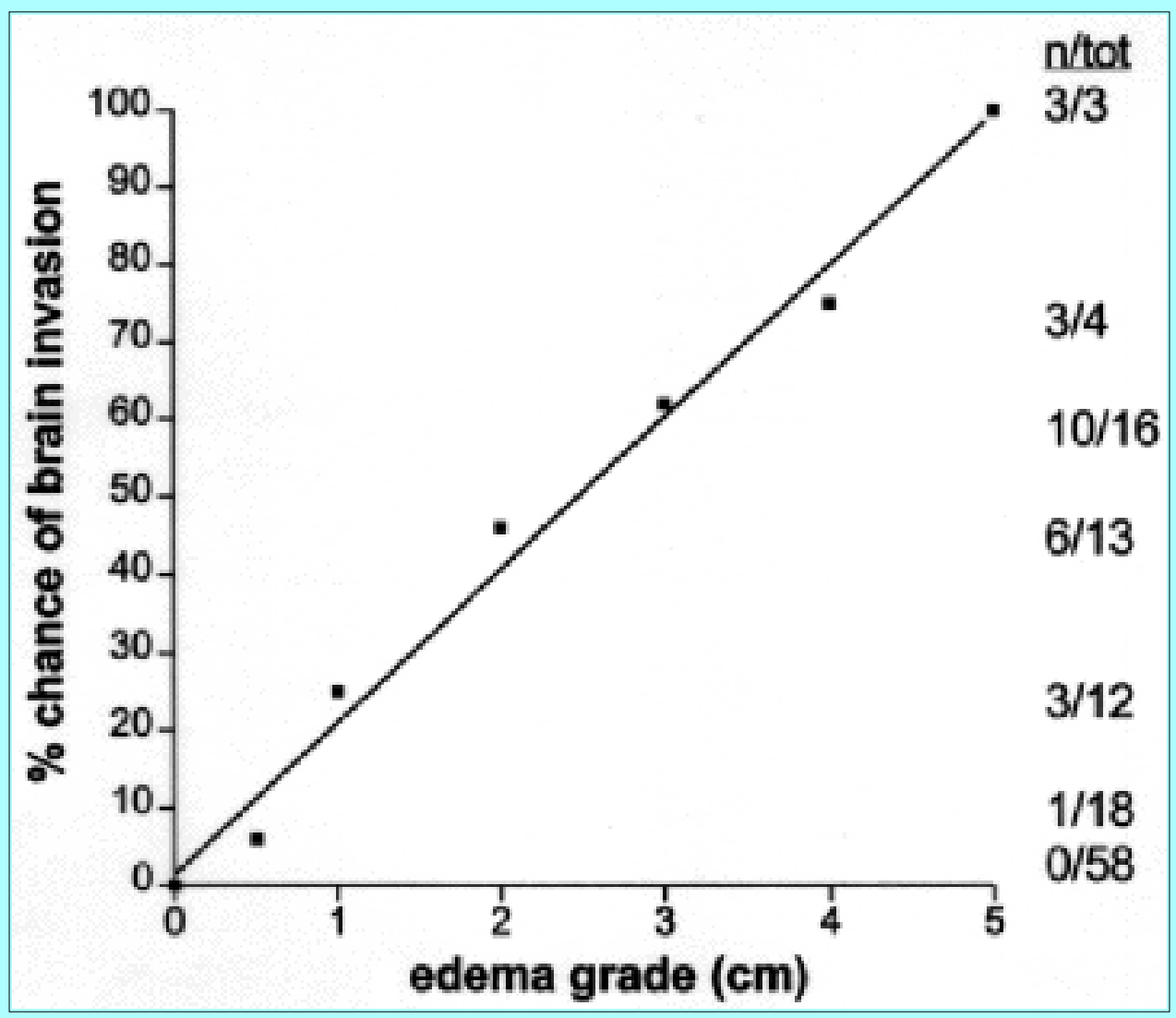

Fig. 5. Graph showing how peritumoral edema grade correlates with the percentage chance of brain invasion by meningiomas. "Chance" refers to the proportion of tumors showing invasion. Fractions to the right of the figure denote the number (n) of tumors showing invasion over the total number (tot) of tumors in each grade. Spearman's rank correlation coefficient $\left(\mathrm{r}_{\mathrm{s}}\right)$ equals 1.0, and the probability value is less than 0.00001 . Note that the chance of invasion increases by $20 \%$ for each centimeter of edema.

The peritumoral edema grade correlated with tumor recurrence after complete resection. An excellent fit to the data was obtained using the following cubic regression equation: percentage chance of recurrence $=(\text { edema grade })^{3}$ X $0.7\left(r_{\mathrm{s}}=1.00, \mathrm{p}<0.0001 ;\right.$ Fig. 6$)$. In all cases in which tumor recurred after complete resection, brain invasion was also demonstrated. 


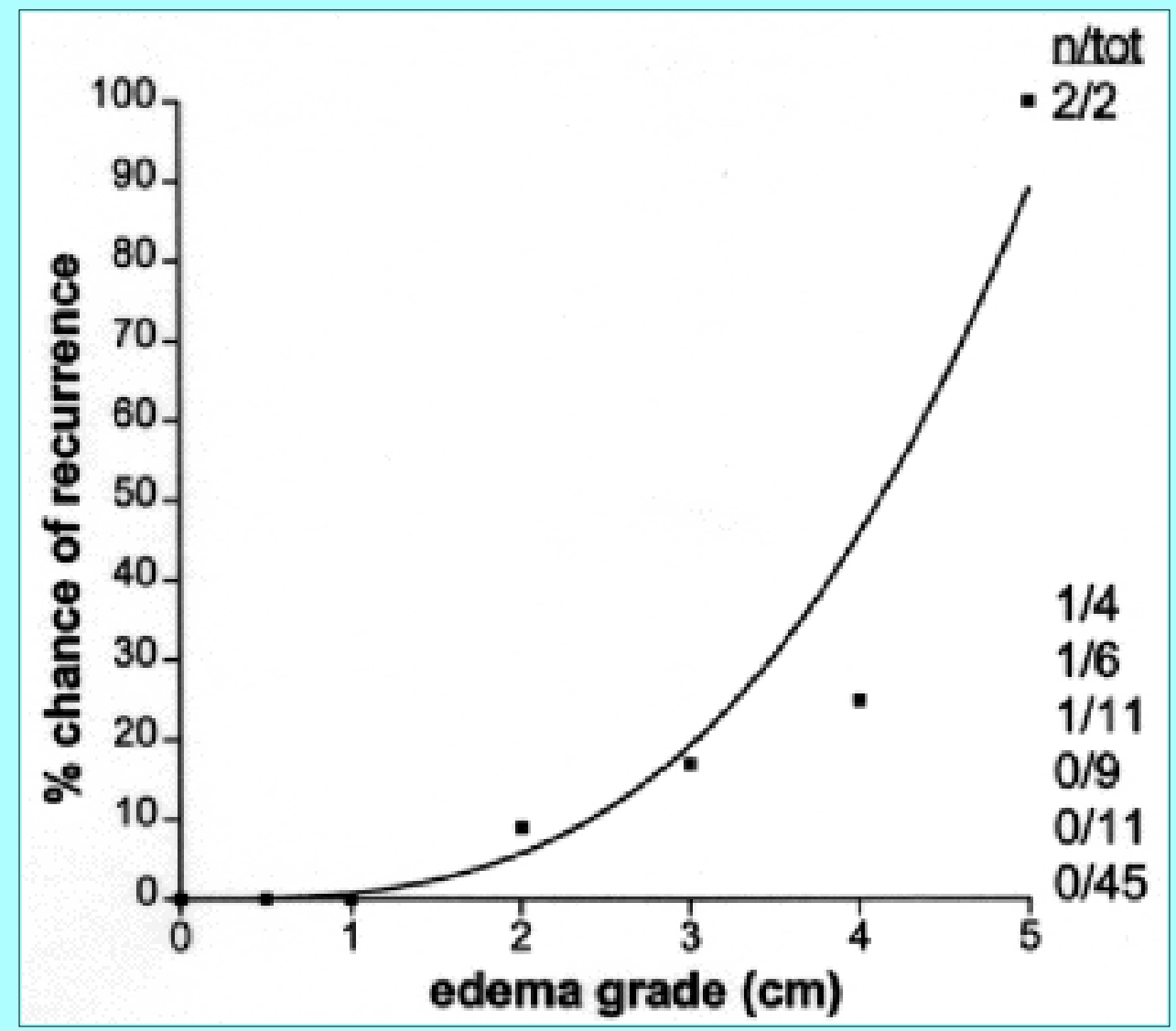

Fig. 6. Graph depicting how peritumoral edema grade correlates with tumor recurrence after complete resection of meningiomas. Fractions to the right of the figure denote the number (n) of tumors that recurred over the total number (tot) of tumors in each grade. Spearman's rank correlation coefficient $\left(r_{s}\right)$ is 0.95 and the probability value is less than 0.0001 . The chance of recurrence increases by approximately $10 \%$ for each edema grade. A more exact fit is obtained by the equation: percentage chance of recurrence $=(\text { edema grade })^{3} \mathrm{X} 0.7$.

Kaplan-Meier plots of recurrence-free time in cases of complete resection compared with those of incomplete resection showed a significant difference in favor of complete resections (Fig. 7). The mean recurrence-free times were $17 \pm 1$ years (standard error of the mean [SEM]) after complete resection and $7 \pm 1$ years $(\mathrm{SEM})$ after incomplete resection. 


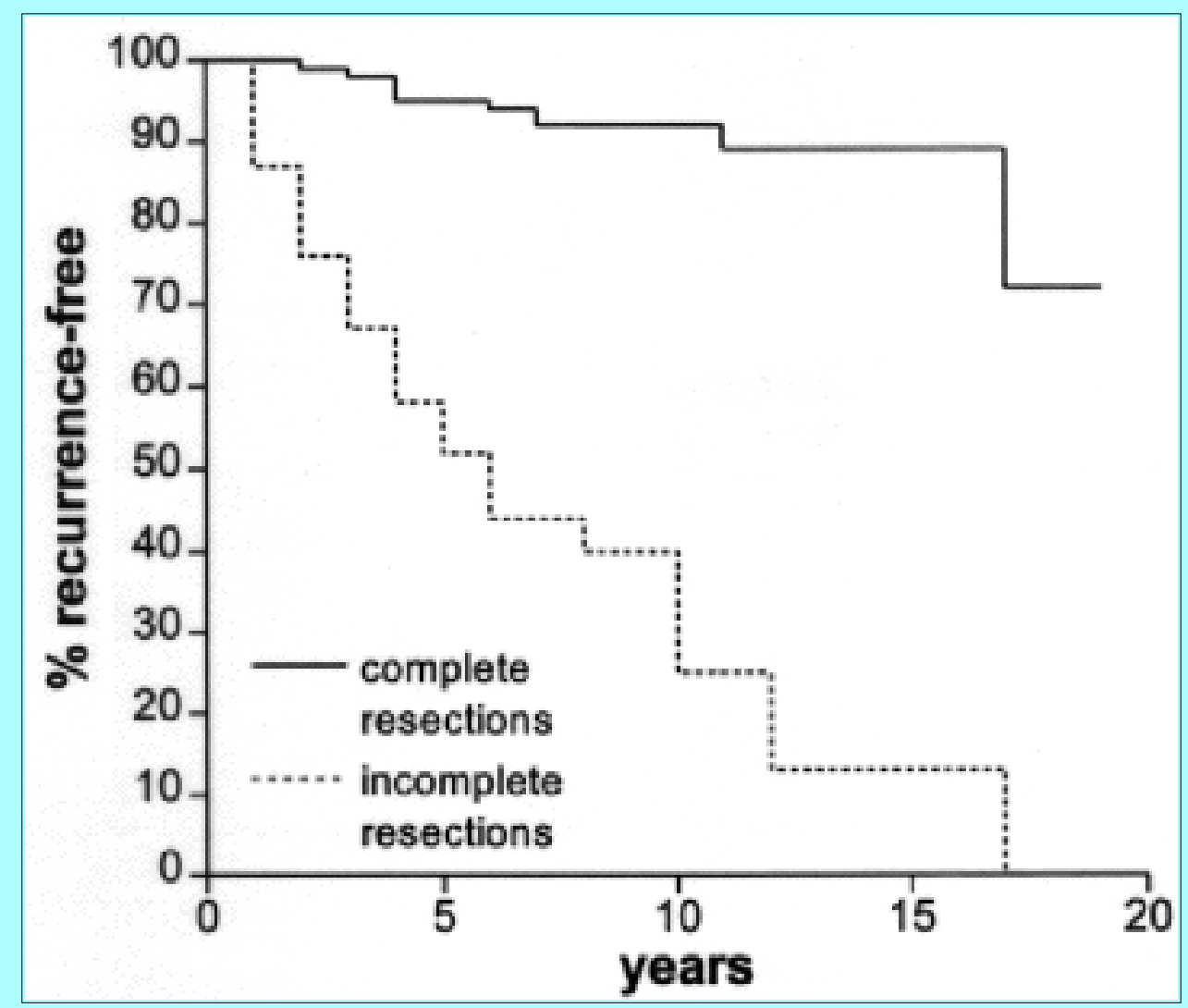

Fig. 7. Kaplan-Meier plots showing the percentages of patients who were recurrence free over time in populations with complete resections and incomplete resections $(\mathrm{p}<0.05)$.

All cases without peritumoral edema in which complete resections of tumor were achieved were free from recurrence for up to 18 years. Cases with peritumoral edema in which complete resections were achieved showed a decline in the recurrence-free time: mean $17 \pm 1$ years (SEM) (Fig. 8). Superimposed Kaplan-Meier plots of recurrence-free time for edema Grades 0 through 5 show increasingly negative slopes of the recurrence-free time curves with increasing edema grade (Fig. 9). 


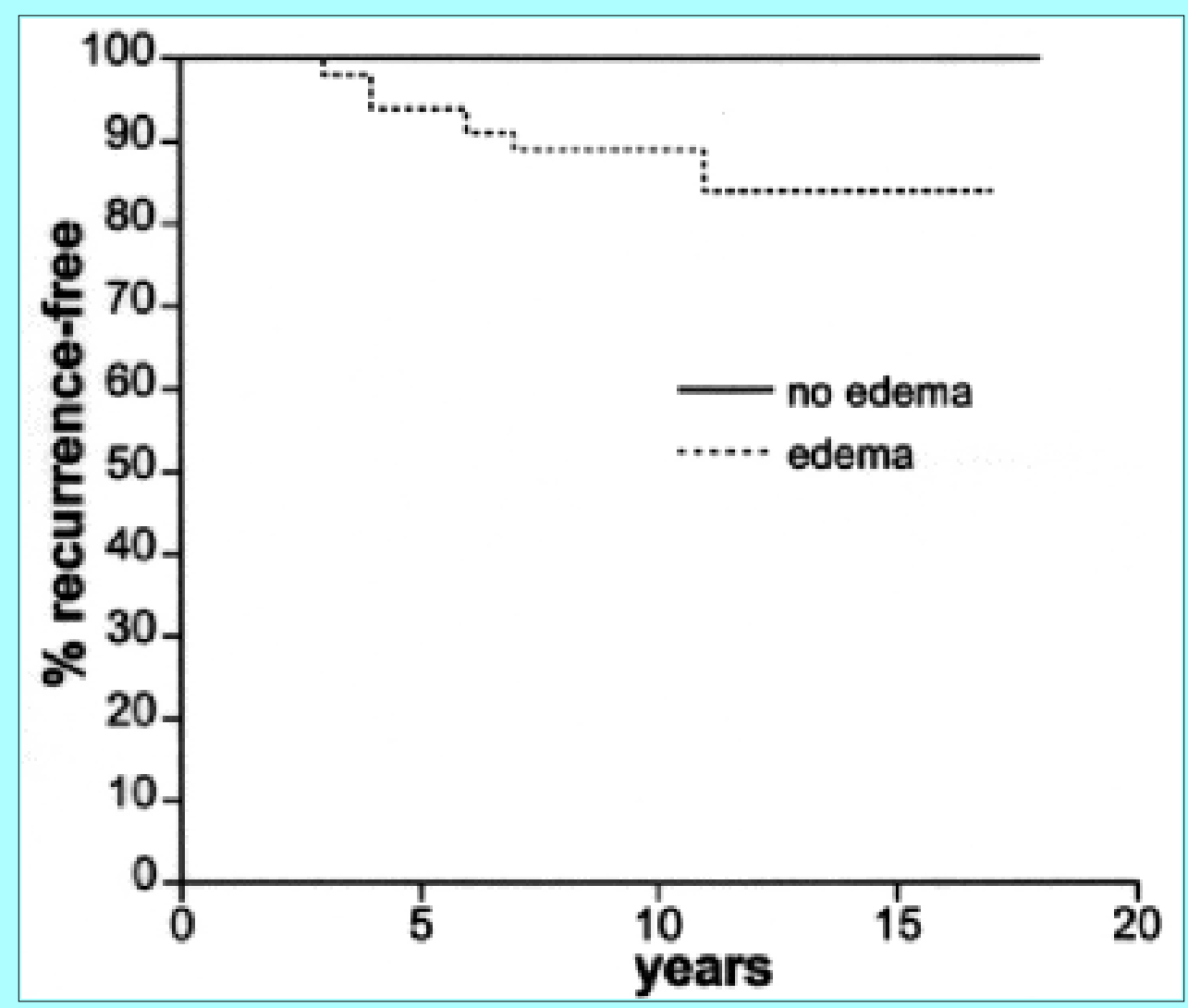

Fig. 8. Kaplan-Meier plots showing the percentages of patients who were recurrence free after complete resections over time in populations with and without peritumoral edema. One hundred percent of cases of complete resection without edema were recurrence free for the entire follow-up period.

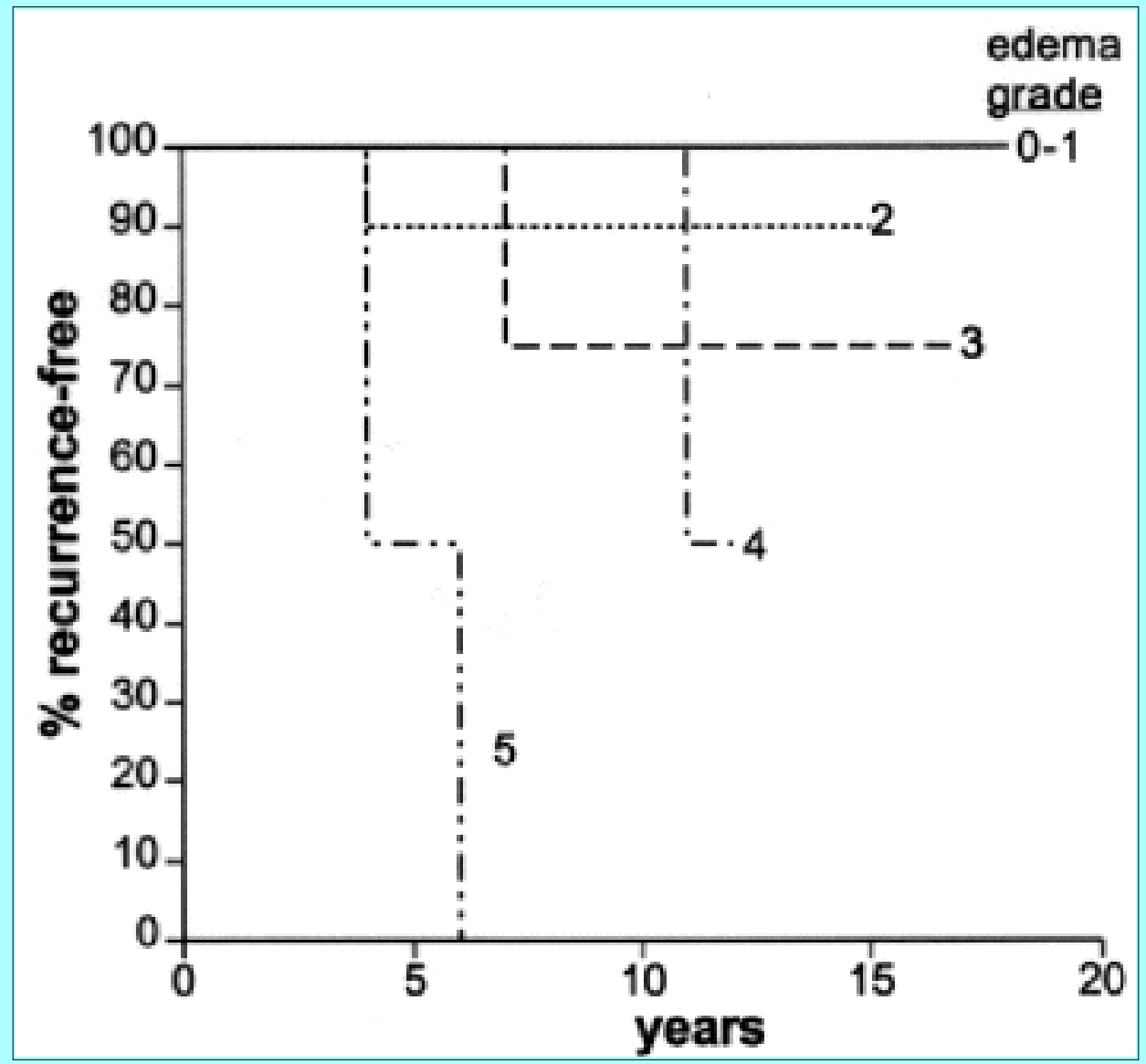


Fig. 9. Kaplan-Meier plots showing the percentages of patients who were recurrence free after complete resection over time. A separate plot for each edema grade is given. Note that the slope is increasingly negative with increasing edema grade.

On the basis of the difference between our mean follow-up time ( $9 \pm 4$ years [SD]) and the mean time to recurrence $(5 \pm 4$ years [SD]), we estimated that $3 \%$ of recurrences in our series may have been missed because the follow-up time was insufficient. If we neglect these, a logistic regression, which requires a nontime-dependent outcome, may be used. We constructed a logistic regression model (forward stepwise conditional) in which patient gender and age at time of resection, completeness of resection, edema grade, brain invasion, tumor volume, histological subtype, left or right side, and location were used as predictors of recurrence. The model correctly predicted $78 \%$ of recurrences. Complete resection $(\mathrm{p}<$ $0.00001)$ and edema grade $(\mathrm{p}=0.02)$ were significant independent predictors of recurrence, whereas brain invasion was not quite significant at the 0.05 level $(\mathrm{p}=0.1)$. The other variables showed probability values far above 0.05 with location as the overall least significant predictor $(\mathrm{p}=0.86$; Table 2$)$.

\begin{tabular}{|c|c|c|c|c|c|c|}
\hline \multicolumn{7}{|c|}{$\begin{array}{c}\text { TABLE } 2 \\
\text { SUMMARY OF MUTMARIATE AND UNMARIATE STATISTICAL AN }\end{array}$} \\
\hline \multirow{2}{*}{$\begin{array}{l}\text { Predictor of } \\
\text { Recurrence }\end{array}$} & \multicolumn{2}{|c|}{$\begin{array}{l}\text { M ultivariate } \\
\text { Logistic } \\
\text { Regession }\end{array}$} & \multicolumn{2}{|c|}{$\begin{array}{l}\text { Univariate } \\
\text { Cox }\end{array}$} & \multicolumn{2}{|c|}{$\begin{array}{c}\text { Multivariate C ox } \\
\text { Proportional } \\
\text { Hazards } \\
\end{array}$} \\
\hline & p value & $r$ Value & p value & $r$ Value & $p$ Value & $r$ Value \\
\hline $\begin{array}{l}\text { complete resection } \\
\text { edema grade } \\
\text { brain invasion } \\
\text { tumor wolume } \\
\text { patient age at resec- } \\
\text { tion }\end{array}$ & $\begin{array}{l}<0.00001 \\
0.02 \\
0.11 \\
0.39 \\
0.47\end{array}$ & $\begin{array}{l}0.6 \\
0.2 \\
0.2 \\
0.0 \\
0.0\end{array}$ & $\begin{array}{l}<0.00001 \\
0.01 \\
0.002 \\
0.26 \\
0.71\end{array}$ & $\begin{array}{l}0.4 \\
0.2 \\
0.2 \\
0.0 \\
0.0\end{array}$ & $\begin{array}{l}<0.00001 \\
0.02 \\
0.02 \\
0.27 \\
0.90\end{array}$ & $\begin{array}{l}0.6 \\
0.1 \\
0.1 \\
0.0 \\
0.0\end{array}$ \\
\hline $\begin{array}{l}\text { histological subtype } \\
\text { It/rt side } \\
\text { patient sex }\end{array}$ & $\begin{array}{l}0.48 \\
0.59 \\
0.62\end{array}$ & $\begin{array}{l}0.0 \\
0.0 \\
0.0\end{array}$ & $\begin{array}{l}0.33 \\
0.41 \\
0.89\end{array}$ & $\begin{array}{l}0.0 \\
0.0 \\
0.0\end{array}$ & $\begin{array}{l}0.07 \\
0.62 \\
0.98\end{array}$ & $\begin{array}{l}0.0 \\
0.0 \\
0.0\end{array}$ \\
\hline tumor location & 0.86 & 0.0 & 0.33 & 0.0 & 0.87 & 0.0 \\
\hline
\end{tabular}

Time-dependent analysis with single and multiple variables was performed using Cox methodologies. In univariate analysis, completeness of resection ( $<0.00001)$, edema grade $(p=0.01)$, and brain invasion $(\mathrm{p}=0.002)$ were significant predictors, whereas patient gender and age at time of resection, tumor volume, histological subtype (including malignant subtypes), left or right side, and location were not (Table 2).

Multivariate modeling (forward stepwise conditional log rank) yielded similar results to logistic regression when using the same variables. Completeness of resection was highly significant ( $\mathrm{p}<$ 0.00001 ), whereas edema grade and brain invasion were the next most significant (both $p=0.02$ ). None of the other variables reached significance (Table 2).

Univariate and multivariate analyses were performed after recoding the histological subtype into three groups: benign, atypical, and malignant. After recoding, the histological subtype reached significance in the univariate analysis $(\mathrm{p}=0.03, \mathrm{r}=0.1)$ and remained significant in the multivariate analysis when completeness of resection was left out of the model $(p=0.03)$. When completeness of resection was 
factored in, atypical and malignant subtypes were insignificant predictors of recurrence $(p=0.4, r=0)$.

\section{DISCUSSION}

Peritumoral low signal attenuation around meningiomas on CT scans has been previously established to represent vasogenic cerebral edema of the white matter by using microgravimetric[35] and electron microscopic methods. $[13,30]$ Peritumoral edema originates in the region of the tumor margin and travels by bulk flow through the relatively loosely interconnected fibers of white matter.[16] Peritumoral edema is found in approximately 50\% of meningiomas.[22] Multiple attempts to correlate the occurrence and degree of peritumoral edema with various factors, including patient gender, tumor location, tumor size, vascularity, cellularity, mitotic activity, steroid receptors, secretory activity, blood supply by intracranial vessels, histological subtype (excepting malignant varieties), and occlusion of veins by the tumor, have failed to establish convincing relationships.[1,3,10,12,13,17,20,24-26,28,30,34]

Peritumoral edema has been successfully correlated with the flow cytometric proliferative index in a study of 20 tumors by Crone and coworkers[11] and in a study by Ide, et al.,[19] of 57 tumors in which MIB-1 staining was used. Meningiomas with edema contained significantly higher levels of vascular endothelial growth factor messenger RNA than those without edema in 16 tumors analyzed by Kalkanis and associates[22] and in 18 tumors analyzed by Provias and colleagues.[31] Increased vascular endothelial growth factor detected by immunostaining correlated with the degree of edema in 37 tumors examined by Goldman, et al.[14] The volume of peritumoral edema in 15 meningiomas correlated with tumor plasminogen activator content in a study by Quindlen and Bucher.[32] Hirashima and coworkers[15] found that platelet-activating factor immunostaining correlated with peritumoral edema in 31 patients. The quantity and location of peritumoral edema was associated with induction of pial blood supply by meningiomas in a recent paper by Bitzer and colleagues.[4]

The results of these studies suggest that meningiomas that are associated with peritumoral edema have higher indices of proliferative activity and produce more vasotrophic and vasoactive factors than those that are not.

Studies in which a correlation of meningioma recurrence with predictive variables has been examined have a much longer history in the literature than those in which a correlation with peritumoral edema was sought; this is due to the relatively recent advent of CT scanning, commencing in approximately 1980. Studies in patients in whom preoperative scans and the necessary follow-up times of 20 years maximum and 10 years on average could be obtained have only now become possible.

The best accepted predictor of recurrence is the 1957 Simpson grading system[33] for completeness of resection. This system defines five grades. Grade I specifies resection of the entire tumor, its dural attachment, and any abnormal bone, whereas Grade V involves decompression only. These grades have been validated to some degree, although no investigators have been able to resolve differences in recurrence between all five grades in a single series. Adegbite, et al.,[2] and Boker, et al.,[6] were able to demonstrate a significant difference in recurrence by combining Grades I and II and comparing them with Grades III and IV. Palma and colleagues[29] were able to resolve a difference in recurrence between Grade I and Grades II through V by studying a series of malignant and atypical tumors only.

Kinjo and colleagues[23] claim that a "Grade 0" resection, as proposed by Borovich and coworkers,[7] can reduce recurrence by resecting a 2-cm dural margin around each tumor. None of the 37 histologically benign meningiomas in their study recurred over a mean follow-up duration of $5 \pm 3$ years (SD) (eight 
patients were followed for 9 years). Their follow-up duration was most likely insufficient, however, because the proportion of patients free from recurrence at 11 years was $90 \%$ in our study, which included malignant subtypes and did not involve Grade 0 resection (Fig. 7). The Borovich "neoplastic field" hypothesis, on which Grade 0 resection is based, suggests that recurrences arise from clusters of meningothelial cells, which these investigators found within the dura near meningiomas in 14 selected cases.[8]

Our definition of complete resection differs from Simpson Grade I in that it specifies absence of residual tumor on postoperative CT or MR images and allows for hyperostotic bone that is not grossly invaded or involved by tumor to be left in place. At our institution, the margin of the resected dura is also usually cauterized using a bipolar instrument.

Malignancy according to histological subtyping (World Health Organization Grade II or III) is the second best established predictor of recurrence. These subtypes represent a small proportion of meningiomas (approximately 8\%) and are widely believed to carry significantly higher risk of recurrence and death to the patient. However, in the study conducted by Palma and colleagues,[29] when Grade I completeness of resection was strictly observed in meningiomas resected from the convexity, nine patients with atypical meningiomas were free from recurrence throughout a 10- to 19-year follow-up period, and four patients with malignant meningiomas were free from recurrence during an 11-year follow up. Because these figures are comparable to recurrence-free rates in benign forms of tumor, they raise the possibility that higher recurrence rates for these tumors are related to greater difficulty in complete resection. Tumor location was found by Palma and colleagues to be a significant predictor of recurrence but only when completeness of resection was not strictly controlled.

Jääskeläinen[21] partially controlled for completeness of resection in a study of 657 meningiomas, Grade I or II (in Grade II, the involved dura was coagulated rather than removed), with a mean follow-up period of 5.4 years. He proposed that tumors described as "soft" in the operative notes were more likely to recur. Patient gender and age, tumor size or weight, and relationship of the tumor to venous sinuses, arteries, and cranial nerves were not found to be significant by either Jääskeläinen or Adegbite, et al.[2]

Ide, et al.,[18] observed a significant correlation between shorter tumor cell doubling times in recurrent meningiomas and high proliferating cell nuclear antigen staining.

The most recent paper on meningioma recurrence by Nakasu and associates[27] focuses on a CT scanning-era series of 146 patients with a mean follow-up time of 9.6 years. The MIB-1 index, tumor size, near sinus location, calcification, edema, hyperostosis, lobulated or mushroom shape, and indistinct brain-tumor interface reached significance for recurrence on univariate analysis, whereas only lobulated and mushroom shapes were significant on multivariate analysis. Although this paper provides sound evidence for shape criteria as a predictor of recurrence, these criteria are qualitative and may be difficult to appreciate on standard axial-plane imaging. Completeness of resection was not strictly controlled (Simpson Grades I-III allowed) and may not have been included in the multivariate analysis.

In the present study we have been able to correlate a very simple edema grading system with edema volume, which we measured using CT scans $(r=0.96)$. The radial thickness of peritumoral edema on the axial slice showing the largest cross-sectional area of tumor is measured at a point that is, subjectively, of average thickness. The scale provided on the film was used to scale the measurement in centimeters. Our regression analysis indicated that a good estimate of edema volume in cubic centimeters may be obtained by multiplying the edema grade by 33 . This system avoids the difficulties inherent in previously 
published edema indices[20] that attempt to normalize the volume of edema per cubic centimeter of tumor volume. Because no relationship between tumor size and amount of edema has been reliably demonstrated, such normalization is unfounded.

We initially expected that the volume of edema would vary as a cubed function of the radius, as it would if the shape of the edematous white matter volume approximated a sphere or quartersphere. Because the volume varied linearly, we must infer that the volume of peritumoral edema, or of the white matter within which it is confined, more closely approximates a cylinder or quartercylinder, whose volumes vary linearly with height. The chance of recurrence was best predicted by the cubic equation, percentage chance of recurrence $=(\text { edema grade })^{3} \times 0.7$, which is in the same form as the formula for the volume of a sphere or partial sphere. We infer from this that the chance of recurrence is proportional to the quantity of residual cells arranged in a hemispherical perimeter zone between the tumor surface and the outer margin of edema. Because of its geometric structure, the volume of this zone varies as the cube of the edema thickness.

We demonstrate strong and statistically significant correlations between the volume of peritumoral edema and both brain invasion and recurrence after gross-total resection. Furthermore, we show that the presence of brain invasion is predictive of recurrence (accuracy 83\%). Our results with respect to edema and brain invasion are consistent with a previous study by Bitzer and colleagues[5] of 179 meningiomas examined retrospectively, in which a significant increase in the incidence of edema when infiltration was present was demonstrated.

Patient age and gender and tumor size, location (left or right side), and histological subtype were not found to be significant predictors of recurrence based on univariate Cox analysis and multivariate logistic regression and Cox proportional hazards modeling. Completeness of resection was found to be the most significant predictor of recurrence $(\mathrm{p}<0.00001)$, before edema grade $(\mathrm{p}=0.02)$ and brain invasion $(\mathrm{p}=$ 0.02) on both logistic regression and Cox multivariate analyses. Completeness of resection, edema grade, and brain invasion were also significant on univariate analysis. The strength of completeness of resection in predicting recurrence $(r=0.6)$ was six times that of edema grade $(r=0.1)$ or brain invasion $(r=0.1)$.

When histological subtype was recoded into three groups--benign, atypical, and malignant--it became significant in univariate analysis $(\mathrm{p}=0.03)$ and multivariate analysis without completeness of resection $(\mathrm{p}=0.03)$, but it lost significance in multivariate analysis that included completeness of resection $(\mathrm{p}=$ $0.4)$. We interpret this to mean that histological malignancy is predictive of recurrence but that the source of this effect is through the influence of malignancy on completeness of resection. Hence, the effect disappears when completeness of resection is controlled. Nevertheless, $100 \%$ of malignant forms in our series were associated with peritumoral edema, and malignant forms were overrepresented in the recurrent group (malignant subtypes represented $40 \%$ of recurrences after complete resection compared with $8 \%$ of all cases. These proportions are identical to those in the recent series by Nakasu, et al.[27]

We have observed that tumor cells invade the brain by advancing along vessels that bridge the gap between the tumor surface and the cortex. We propose that these cells are the most frequent source of recurrences after "complete" resection. As bridging vessels enter the cortical surface, they pierce the pia and are invested by it for a short distance, creating the so-called Virchow-Robbins space between the pia and vessel. The pial investiture is then replaced within the brain parenchyma by a circumferential covering of astrocytic foot processes from local astrocytes whose function appears to be to maintain tight junctions between vascular endothelial cells through the secretion of short-range humoral factors. 
Absence of astrocytic foot processes has been shown to cause brain capillary endothelial cells to lose their tight junctions,[37] whereas addition of astrocyte-conditioned medium to a non-central nervous system endothelial cell culture causes them to form tight junctions.[38] As tumor cells enter the plane between the vessel and its covering of astrocytic foot processes, they are expected to disrupt the relationship between the astrocytic foot processes and the vessel endothelium, either through physical interposition or through the interference of vasoactive factors produced by the tumor cells. This would cause opening of the tight junctions and edema by bulk-flow fluid exudation.[16,36]

We can infer from our data a causal relationship between brain invasion, quantity of peritumoral edema, and recurrence after complete resection. Our evidence for causality is at the human prospective cohort study level with evidence of a dose-dependent gradient and the correct temporal sequence. Furthermore, the criterion of biological plausibility is satisfied by the direct correlation observed between the quantity of edema and the risk of recurrence after gross-total resection. Current neurosurgical techniques for the excision of meningiomas involve meticulous sparing of brain tissue in the tumor bed, which will tend to leave behind tumor cells that have invaded the brain if any are present.

\section{CONCLUSIONS}

Our results indicate that evidence of peritumoral edema on CT scans can be used by the neurosurgeon to predict the chances of brain invasion and recurrence preoperatively and noninvasively. A simple grading system based on the subjectively determined average thickness of edema seen on the best axial CT slice was highly correlated with edema volume determined by digitizing the scans (edema volume in cubic centimeters $=33 \mathrm{X}$ edema grade (in centimeters) $[\mathrm{r}=0.96 ; 29$ cases $]$ ). The chance of finding brain invasion on microscopic examination increased by $20 \%$ per centimeter of edema thickness on axial CT scans $\left(\mathrm{r}_{\mathrm{s}}=1, \mathrm{p}<0.0001 ; 124\right.$ cases). Histopathological documentation of brain invasion was predictive of recurrence (accuracy 83\%, sensitivity 89\%, specificity 82\%, PPV 36\%, NPV 99\%, odds ratio 36). The chance of recurrence after complete resection was given by the regression equation: percentage chance of recurrence $=(\text { edema grade })^{3} \times 0.7$, which can be used as a rule to predict the chance of recurrence on the basis of CT scans $\left(\mathrm{r}_{\mathrm{s}}=1.00, \mathrm{p}<0.0001 ; 86\right.$ cases $)$. A patient with 3 -cm-thick peritumoral edema on preoperative CT scanning, for example, has a $19 \%$ chance of recurrence within 10 years if a gross-total resection is achieved. The probabilities given by this equation for edema Grades 1, 2, 3, 4, and $5 \mathrm{~cm}$ are $1,6,19,45$, and $88 \%$, respectively. The significance of edema grade and brain invasion was confirmed using univariate and multivariate time-dependent modeling and logistic regression. Completeness of resection was the most powerful predictor $(\mathrm{p}<0.00001, \mathrm{r}=0.6)$, followed by edema grade on CT scanning $(\mathrm{p}=0.02, \mathrm{r}=0.1)$ and brain invasion on histological specimen $(\mathrm{p}=0.02, \mathrm{r}=0.1)$. Atypical and malignant subtypes were found to recur more frequently, possibly through the influence of malignancy on completeness of resection. Other subtypes, patient age and gender and tumor volume and location were not found to be significant predictors of recurrence.

We recommend that neuroradiologists report edema grade and that neuropathologists examine specimens for brain invasion by the methods indicated here. These relationships may be useful not only for obtaining better prognostic information for the patient but also for the neurosurgeon in the operating room, who might consider providing a resection margin around tumors associated with edema measuring more than $1 \mathrm{~cm}$. Future studies on meningioma recurrence should strictly control for completeness of resection as defined here to obtain meaningful results with respect to less powerful predictors. Follow-up times of 20 years maximum and 10 years on average are recommended. 


\section{References}

1. Abe T, Black PM, Ojemann RG, et al: Cerebral edema in intracranial meningiomas: evidence for local and diffuse patterns and factors associated with its occurrence. Surg Neurol 42:471-475, 1994

2. Adegbite AB, Khan MI, Paine KWE, et al: The recurrence of intracranial meningiomas after surgical treatment. J Neurosurg 58:51-56, 1983

3. Bitzer M, Topka H, Morgalla M, et al: Tumor-related venous obstruction and development of peritumoral brain edema in meningiomas. Neurosurgery 42:730-737, 1998

4. Bitzer M, Wöckel L, Luft AR, et al: The importance of pial blood supply to the development of peritumoral brain edema in meningiomas. J Neurosurg 87:368-373, 1997

5. Bitzer M, Wöckel L, Morgalla M, et al: Peritumoural brain oedema in intracranial meningiomas: influence of tumour size, location and histology. Acta Neurochir 139:1136-1142, 1997

6. Boker DK, Meurer H, Gullotta F: Recurring intracranial meningiomas. Evaluation of some factors predisposing for tumor recurrence. J Neurosurg Sci 29:11-17, 1985

7. Borovich B, Doron Y: Recurrence of intracranial meningiomas: the role played by regional multicentricity. J Neurosurg 64:58-63, 1986

8. Borovich B, Doron Y, Braun J, et al: Recurrence of intracranial meningiomas: the role played by regional multicentricity. Part 2. Clinical and radiological aspects. J Neurosurg 65:168-171, 1986

9. Chan RC, Thompson GB: Morbidity, mortality, and quality of life following surgery for intracranial meningiomas. A retrospective study in 257 cases. J Neurosurg 60:52-60, 1984

10. Constantini S, Tamir J, Gomori MJ, et al: Tumor prostaglandin levels correlate with edema around supratentorial meningiomas. Neurosurgery 33:204-211, 1993

11. Crone KR, Challa VR, Kute TE, et al: Relationship between flow cytometric features and clinical behavior of meningiomas. Neurosurgery 23:720-724, 1988

12. de Vries J, Wakhloo AK: Cerebral oedema associated with WHO-I, WHO-II, and WHO-III-meningiomas: correlation of clinical, computed tomographic, operative and histological findings. Acta Neurochir 125:34-40, 1993

13. Gilbert JJ, Paulseth JE, Coates RK, et al: Cerebral edema associated with meningiomas. Neurosurgery 12:599-605, 1983

14. Goldman CK, Bharara S, Palmer CA, et al: Brain edema in meningiomas is associated with increased vascular endothelial growth factor expression. Neurosurgery 40:1269-1277, 1997

15. Hirashima Y, Hayashi N, Fukuda O, et al: Platelet-activating factor and edema surrounding meningiomas. J Neurosurg 88:304-307, 1998

16. Hossman KA, Blöink M, Wilmes J, et al: Experimental peritumoral edema of the cat brain. Adv Neurol 28:323-340, 1980

17. Ide M, Jimbo M, Kubo O, et al: Peritumoral brain edema associated with meningioma--histological 
study of the tumor margin and surrounding brain. Neurol Med Chir 32:65-71, 1992

18. Ide M, Jimbo M, Yamamoto M, et al: Growth rate of intracranial meningioma: tumor doubling time and proliferating cell nuclear antigen staining index. Neurol Med Chir 35:289-293

19. Ide M, Jimbo M, Yamamoto M, et al: MIB-1 staining index and peritumoral brain edema of meningiomas. Cancer 78:133-143, 1996

20. Inamura T, Nishio S, Takeshita I, et al: Peritumoral brain edema in meningiomas--influence of vascular supply on its development. Neurosurgery 31:179-185, 1992

21. Jääskeläinen J: Seemingly complete removal of histologically benign intracranial meningioma: late recurrence rate and factors predicting recurrence in 657 patients. A multivariate analysis. Surg Neurol 26:461-469, 1986

22. Kalkanis SN, Carroll RS, Zhang J, et al: Correlation of vascular endothelial growth factor messenger RNA expression with peritumoral vasogenic cerebral edema in meningiomas. J Neurosurg 85:1095-1101, 1996

23. Kinjo T, Al-Mefty O, Kanaan I: Grade zero removal of supratentorial convexity meningiomas. Neurosurgery 33:394-399, 1993

24. Lobato RD, Alday R, Gómez PA, et al: Brain oedema in patients with intracranial meningioma. Correlation between clinical, radiological, and histological factors and the presence and intensity of oedema. Acta Neurochir 138:485-493, 1996

25. Maiuri F, Gangemi M, Cirillo S, et al: Cerebral edema associated with meningiomas. Surg Neurol 27:64-68, 1987

26. Maiuri F, Montagnani S, Iaconetta G, et al: Correlation between sex hormone receptors and peritumoral edema in intracranial meningiomas. J Neurosurg Sci 38:29-33, 1994

27. Nakasu S, Nakasu Y, Nakajima M, et al: Preoperative identification of meningiomas that are highly likely to recur. J Neurosurg 90:455-462, 1999

28. Ohno K, Matsushima Y, Aoyagi M, et al: Peritumoral cerebral edema in meningiomas: the role of the tumor-brain interface. Clin Neurol Neurosurg 94:291-295, 1992

29. Palma L, Celli P, Franco C, et al: Long-term prognosis for atypical and malignant meningiomas: a study of 71 surgical cases. J Neurosurg 86:793-800, 1997

30. Philippon J, Foncin JF, Grob R, et al: Cerebral edema associated with meningiomas: possible role of a secretory-excretory phenomenon. Neurosurgery 14:295-301, 1984

31. Provias J, Claffey K, delAguila L, et al: Meningiomas: role of vascular endothelial growth factor/vascular permeability factor in angiogenesis and peritumoral edema. Neurosurgery 40:1016-1026, 1997

32. Quindlen EA, Bucher AP: Correlation of tumor plasminogen activator with peritumoral cerebral edema. A CT and biochemical study. J Neurosurg 66:729-733, 1987 
33. Simpson D: The recurrence of intracranial meningiomas after surgical treatment. J Neurol Neurosurg Psychiatry 20:22-39, 1957

34. Smith HP, Challa VR, Moody DM, et al: Biological features of meningiomas that determine the production of cerebra edema. Neurosurgery 8:428-433, 1981

35. Takagi H, Shapiro K, Marmarou A, et al: Microgravimetric analysis of human brain tissue. Correlation with computerized tomography scanning. J Neurosurg 54:797-801, 1981

36. Vaz R, Borges N, Cruz C, et al: Cerebral edema associated with meningiomas: the role of peritumoral brain tissue. J Neurooncol 36:285-291, 1998

37. Wolburg H, Neuhaus J, Kniesel U, et al: Modulation of tight junction structure in blood-brain barrier endothelial cells. Effects of tissue culture, second messengers and cocultured astrocytes. J Cell Sci 107:1347-1357, 1994

38. Yamagata K, Tagami M, Nara Y, et al: Astrocyte-conditioned medium induces blood-brain barrier properties in endothelial cells. Clin Exp Pharmacol Physiol 24:710-713, 1997

Manuscript received November 16, 1998.

Accepted in final form May 10, 1999.

This study was presented in part at the annual meetings of the Canadian Association of Neuropathologists, in Ottawa, Ontario, Canada, September 1984, and in Saskatoon, Saskatchewan, Canada, October 1998.

Address correspondence to: Boleslaw Lach, M.D., Ph.D., Department of Neuropathology, Laboratory Medicine Building, Ottawa Hospital, Civic Campus, 1053 Carling Avenue, Ottawa, Ontario, K1Y 4E9 Canada.email: blach@civich.ottawa.on.ca. 